\title{
Functional recovery from chronic writer's cramp by brain-computer interface rehabilitation: a case report
}

\author{
Yasunari Hashimoto ${ }^{*}$, Tetsuo Ota ${ }^{2}$, Masahiko Mukaino ${ }^{3}$, Meigen Liư ${ }^{4}$ and Junichi Ushiba ${ }^{5}$
}

\begin{abstract}
Background: Dystonia is often currently treated with botulinum toxin injections to spastic muscles, or deep brain stimulation to the basal ganglia. In addition to these pharmacological or neurosurgical measures, a new noninvasive treatment concept, functional modulation using a brain-computer interface, was tested for feasibility. We recorded electroencephalograms (EEGs) over the bilateral sensorimotor cortex from a patient suffering from chronic writer's cramp. The patient was asked to suppress an exaggerated beta frequency component in the EEG during hand extension.

Results: The patient completed biweekly one-hour training for 5 months without any adverse effects. Significant decrease of the beta frequency component during handwriting was confirmed, and was associated with clear functional improvement.

Conclusion: The current pilot study suggests that a brain-computer Interface can give explicit feedback of ongoing cortical excitability to patients with dystonia and allow them to suppress exaggerated neural activity, resulting in functional recovery.
\end{abstract}

Keywords: Neurorehabilitation, Neurofeedback, Upper extremity, Motor learning, Cortico-muscular coherence

\section{Background}

Brain-Computer Interface (BCI) technology has already been successfully used to control a computer mouse cursor and a robotic arm by thought, and thus has been expected to become a tool to compensate for lost motor functions in patients with spinal cord injury [1] or amyotrophic lateral sclerosis [2]. Recently, some research groups succeeded in showing another possible use of $\mathrm{BCI}$, that is, as a tool to promote neural plasticity causing functional recovery from stroke [3-9]. The number of clinical applications of such BCI-based neurorehabilitation is expected to increase in the near future.

Focal dystonia is a disorder of movement characterized by involuntary, sustained muscle contractions, frequently causing twisting and repetitive movements or abnormal postures of a body part [10]. Writer's cramp (WC) is an example of task-specific focal hand dystonia. WC was

\footnotetext{
* Correspondence: hashimya@mail.kitami-it.ac.jp

${ }^{1}$ Department of Electrical and Electronic Engineering, Kitami Institute of Technology, Hokkaido, Japan

Full list of author information is available at the end of the article
}

once believed to be a purely psychological problem, but more recently is understood to be due to more specific neural dysfunction, including that of the basal ganglia $[11,12]$. Disinhibition and overexcitation of the corticobasal ganglia-thalamic loop may lead to co-contractions and dystonic postures [13].

A recent transcranial magnetic stimulation study has also revealed that there is shift in the balance between excitation and inhibition in local circuits of the primary motor cortex in focal dystonia [13]. Sensory evoked potential recordings in focal dystonia (and in other types of dystonic disorders) also indicate that in dystonia there is impaired inhibition at spinal and cortical levels of the somatosensory system, which can lead to an abnormal sensory assistance to ongoing motor programs, resulting in motor abnormalities [14].

Toro et al. investigated abnormal activity at the cortical level with a scalp electroencephalogram [15]. In their results, a significantly lower amount of eventrelated desynchronization (ERD) in the $20-30 \mathrm{~Hz}$ band was found in dystonic patients over the contralateral 
and midline centroparietal regions, from $-100 \mathrm{~ms}$ to $+200 \mathrm{~ms}$ with respect to electromyogram (EMG) onset. If we could provide explicit online representation of such dystonic-specific EEG features, it could help WC patients to voluntarily return the cortical state to normal using BCI neurofeedback.

To prove its concept, the present study employed a BCI paradigm to provide visual feedback of ongoing EEG features that represents the exaggerated excitability of the sensorimotor cortex during hand movement (Figure 1), and assessed neurological and behavioral changes through 5-month use in a WC patient as a clinical pilot study ${ }^{\mathrm{a}}$.

\section{Results and discussion}

After 10 BCI training sessions, the WC participant clearly showed a reduction of dystonic movement during writing, and their handwriting of Japanese characters showed a reduction in distortion (Figure 2). The patient also showed some reduction in hand rigidity during writing.

For further analysis, EEG over the bilateral sensorimotor cortex and EMG from the dystonic right extensor carpi radialis (ECR) were recorded during tonic wrist extension, and the power spectra and coherency of the EEG signals and the rectified EMG signal were calculated (Figures 3 and 4). In the pre-training condition (Figure 3), the coherence peak achieved 0.12 between the left EEG and the rectified EMG. A coherence peak was also found between the right EEG and the rectified EMG. The frequency band of significant coherence expanded from $26 \mathrm{~Hz}$ to $34 \mathrm{~Hz}$ both in the left EEG-EMG coherence (peak value at $33 \mathrm{~Hz}=0.12$ ) and in the right EEG-EMG coherence (peak value at $33 \mathrm{~Hz}=0.10$ ). In contrast, the post-training condition data showed no significant

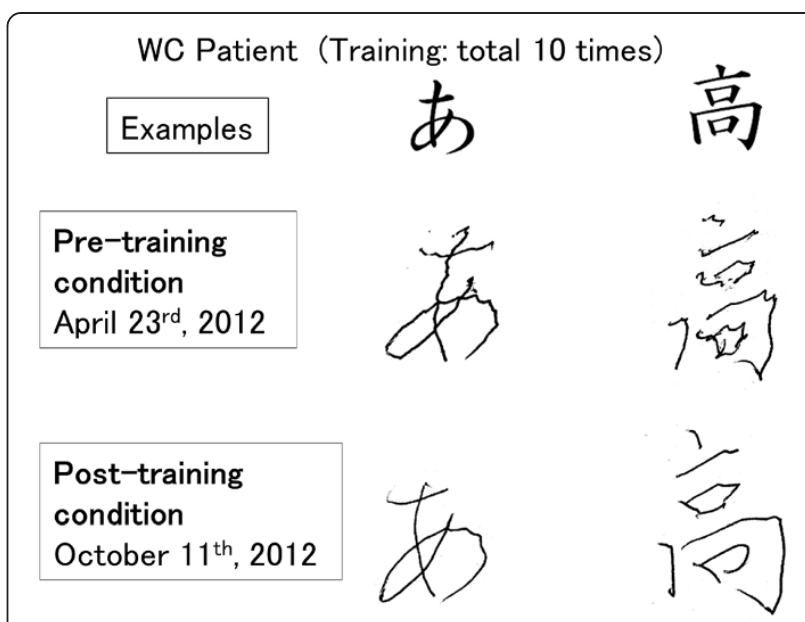

Figure 2 An example of Japanese and Chinese characters and the handwriting of the patient (Pre-training and post-training condition).

coherence between the left EEG-EMG, and a low peak (peak value at $28 \mathrm{~Hz}=0.03$ ) in right EEG-EMG coherence.

In the ERD/ event-related synchronization (ERS) analysis for EEG data during a repetitive motor task, significant extension-related EEG power increase (ERS) in the beta band was observed bilaterally in the pre-training condition (Figure 5A-B; significance level =99\%). The frequency bands of this ERS expanded from $26 \mathrm{~Hz}$ to $30 \mathrm{~Hz}$ in the left EEG and from $27 \mathrm{~Hz}$ to $32 \mathrm{~Hz}$ in the right EEG. Both frequency bands were within the frequency band of dystonia-specific EEG features determined by the coherence analysis. After BCI training, the averaged ERS decreased bilaterally from $67 \%$ to $28 \%$ in the left EEG (in $26-30 \mathrm{~Hz}$, Figure $5 \mathrm{E}$ ) and from $62 \%$ to $32 \%$ in the right EEG (in 27-32 Hz, Figure 5F).

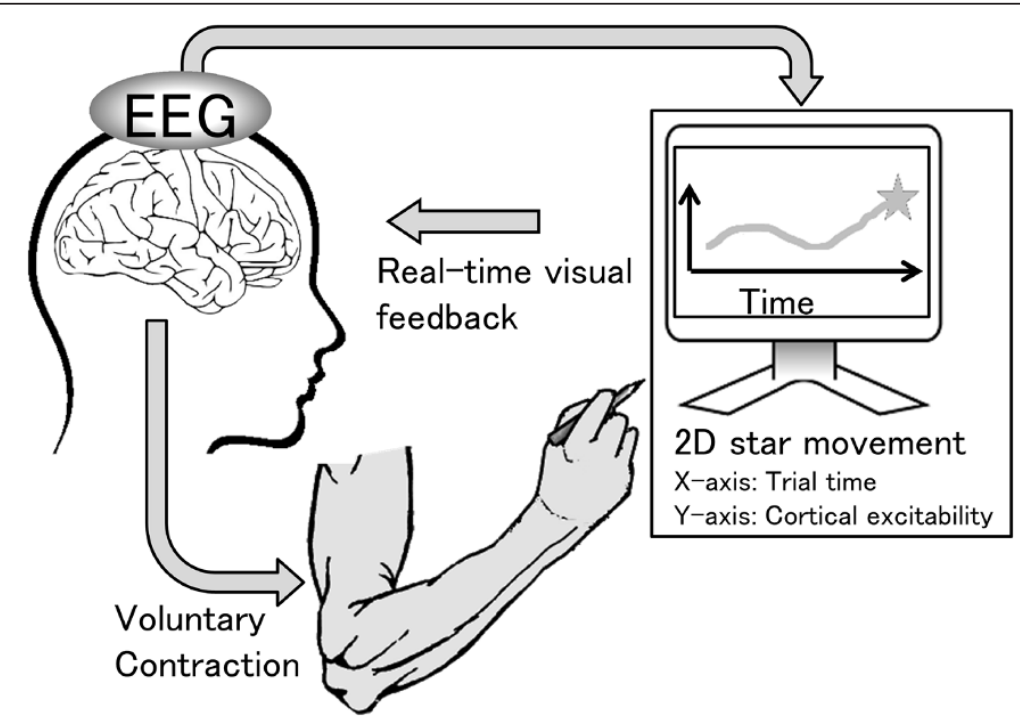

Figure 1 Schematic diagram of the brain-computer interface that feeds back information about cortical excitability in the sensorimotor cortex. 

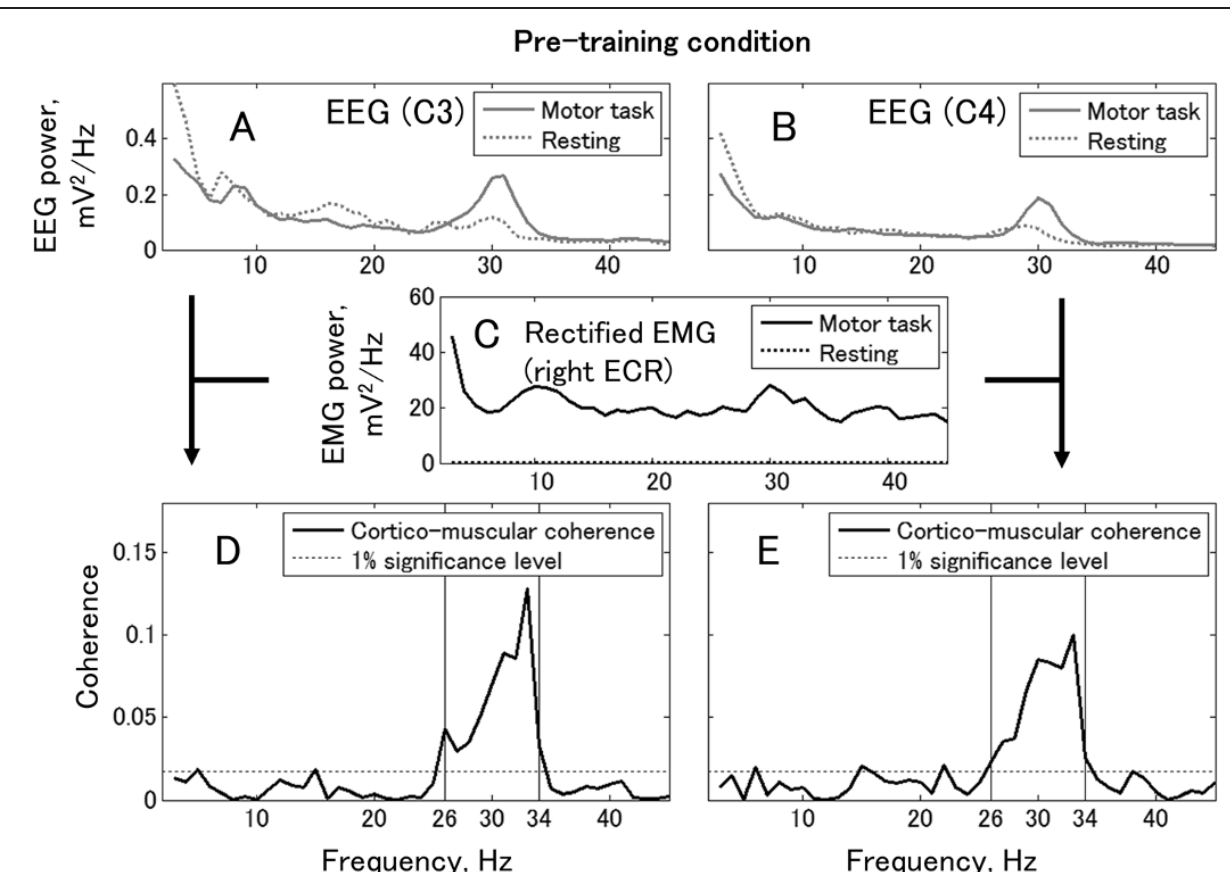

Figure 3 Cortico-muscular coherence and power spectra of EEG and rectified EMG in pre-training condition. Top row shows EEG power spectra from the left EEG (Panel A) and the right EEG (Panel B). Middle row shows the power spectrum of rectified EMG recorded from the right ECR muscle (Panel C). Bottom row shows coherence between EEG and rectified EMG of the right ECR. The horizontal lines denote the $99 \%$ confidence limit. Panels $\mathbf{D}$ and $\mathbf{E}$ show the Laplacian EEG from the left and right hemispheres, respectively.

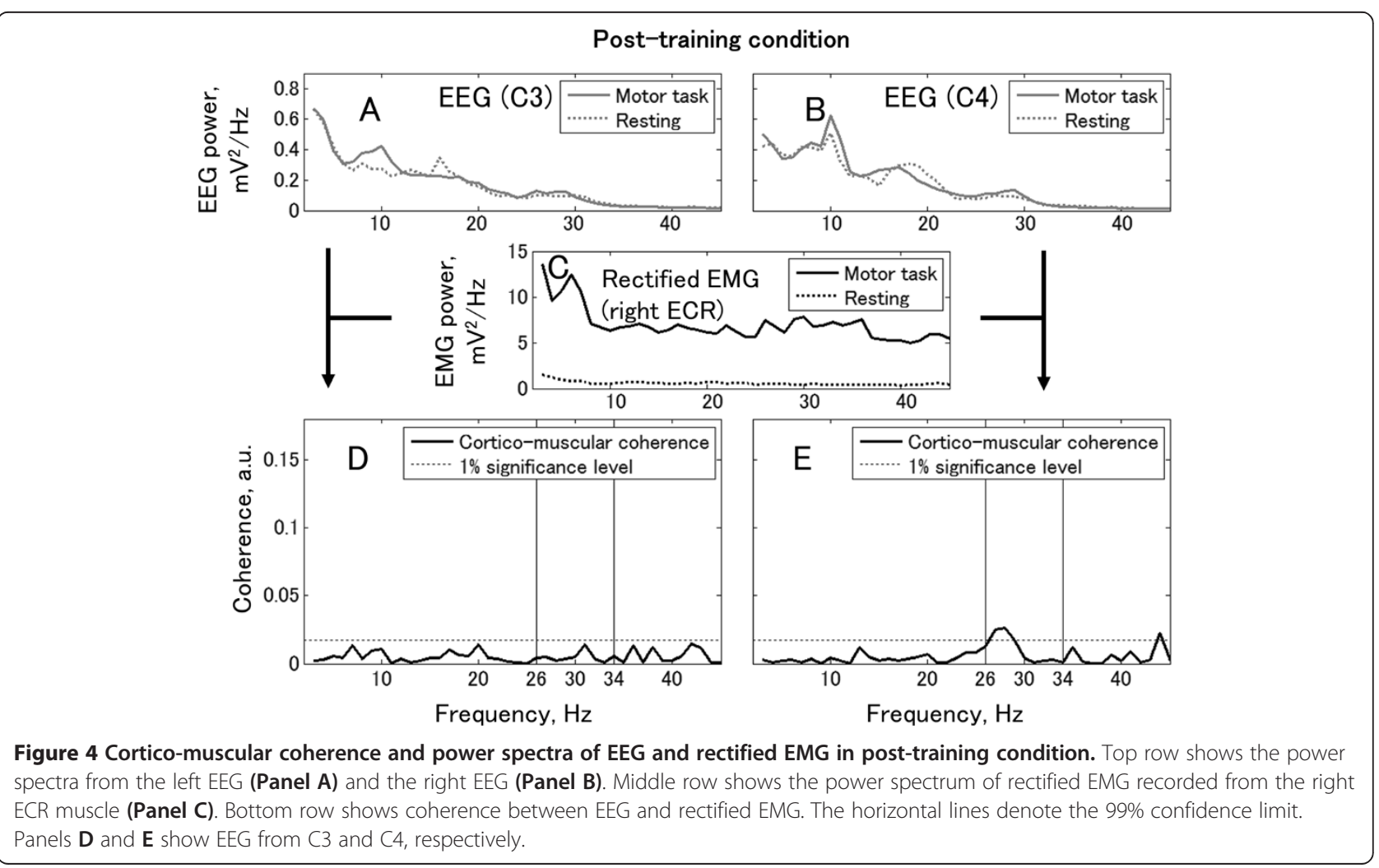




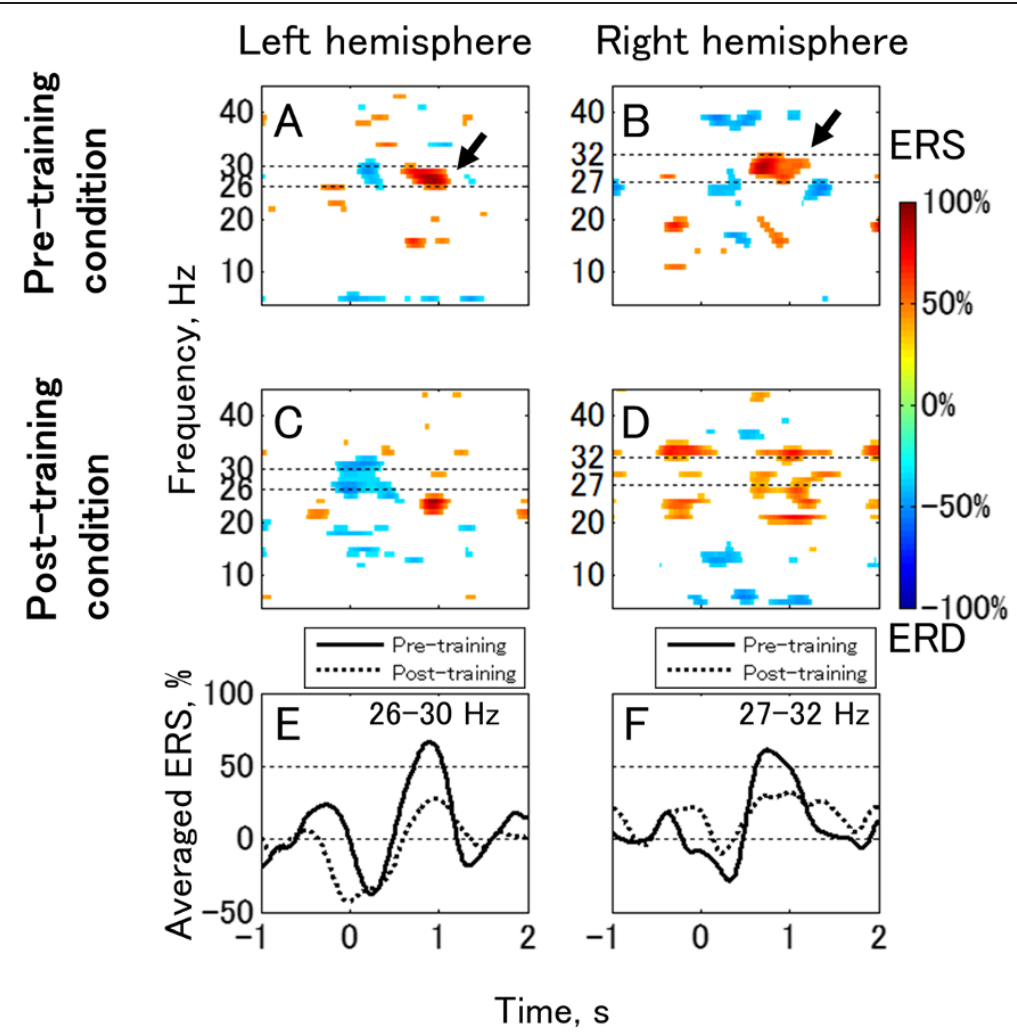

Figure $\mathbf{5}$ ERD/ERS produced by repetitive motor task. Panels $\mathbf{A}$ and $\mathbf{B}$ are computed from data recorded prior to training and Panels $\mathbf{C}$ and $\mathbf{D}$ are from after training. The lower panels show averaged ERD/ERS values in the $26-30 \mathrm{~Hz}$ band (Panel E) and in the $27-32 \mathrm{~Hz}$ band (Panel F) for comparison between pre- and post-training conditions. The left column (Panels A, C, and E) and the right column (Panels B, D, and F) represent the data recorded from around C3 (left hemisphere) and C4 (right hemisphere), respectively. Statistically non-significant areas are shown as blank in Panels A-D. The significance level is set to $\mathrm{P}<0.01$.

Certainly, there is some evidence for the involvement of the bilateral motor cortices in the abnormal muscle activity in focal dystonia. Tecchio et al. reported patients' exaggerated and bilaterally coupled cortico-muscular coherence in the beta band (mean frequency $21.5 \mathrm{~Hz}$ ) during isometric contraction [16], which is not seen in healthy participants (e.g. [17]). These studies suggest the existence of different mechanisms of cortico-muscular coupling from healthy individuals in dystonia patients. We found a large beta ERS from the hand motor areas in both hemispheres in the pre-training condition (Figure 5). Since such EEG characteristics were consistent with previous coherence studies [18], it is natural to assume that large ERS is observed in the frequency in which large cortico-muscular coherence exists. Therefore, we consider that selecting the 26-34 Hz EEG amplitude to represent dystonia-specific EEG features was reasonable.

Cortico-muscular coherence provides a quantitative measure of the linear components of the coupling between EEG and rectified EMG. It should be noted that the coherence is not expected to be a direct estimate of the anatomical neural connections. Tecchio et al. argue that high cortico-muscular coherence found in dystonic patients should be understood in a physiological sense as signs of a different (abnormal) sensorimotor integration [16]. They also suggest that healthy subjects transiently mediate movement control by tuning their neuronal firing rates more than by cortico-muscular synchronization, and that the repertoire of voluntary motor control strategies is reduced in dystonic patients. We agree with this suggestion and the improvement of writing after BCI training (Figure 2) can be explained by a change in the motor control strategy for writing. The cortico-muscular coherence might quantitatively indicate this change from synchronization leading control to other modes, for example, firing rate tuning.

Our result clearly shows a decrease of ERS in the beta frequency band associated with a significant improvement of handwriting after biweekly BCI training. Since the ERS in this beta frequency band must be a dystoniarelated feature, providing visual feedback of the ongoing ERS level through our BCI system might promote plastic and functional reorganization in the neural network of this patient. Considering that it has been over 5 years since the appearance of symptoms, and that conventional 
treatments were not effective for this patient, BCI training might have different applications for functional cortical modulation than previously thought.

In addition, in the current study, clinical scales were not measured before or after the intervention. Instead, the handwriting on a paper was employed, and the authors set the main focus of the study on EEG pattern changes induced by our BCI system in a WC patient. Therefore, degree of the functional improvement of the patient's handwriting is still unclear in this study. As a next step, it is necessary to quantify the functional improvement using, for example, the Writer's Cramp Rating Scale [19] or other dystonia rating scales. To inquire more details regarding functional improvement of handwriting by trainings, it will need long-term follow-up study. There are still open questions whether more training can change EEG pattern or improve hand writing, or whether the improvement of the handwriting can be sustained after finishing the $\mathrm{BCI}$ training.

\section{Conclusions}

To date, mainstream $\mathrm{BCI}$ rehabilitation has aimed to induce functional reorganization for stroke patients or incomplete spinal cord injury patients [4]. The current study, however, demonstrates another application of therapeutic BCI for patients with exaggerated cortical activity associated with involuntary muscle contractions, such as a dystonia patient, using visual feedback of abnormal EEG activity. Though this study is a single-case study without control participants and is also in the pilot stage, the encouraging results may facilitate further larger scale studies or randomized controlled trials.

\section{Methods}

The WC patient participated biweekly training for 5 months (10 times) with a BCI system that detects dystonic contraction-related EEG features and displays them for the patient. The EEG features were calculated from bilaterally recorded EEG in 26-34 $\mathrm{Hz}$ frequency bands, where significant cortico-muscular coherence was observed during tonic ECR muscle extension in the right (dominant) dystonic hand. During the BCI training, the patient was instructed to repeat hand extension for 70 seconds, and, during extension, keep the amount of dystonia-specific EEG features low level, the same as during resting, using the feedback from the BCI.

\section{Participant}

A patient with the diagnosis of WC (age 67 years; female; 1.5 years from WC onset) participated in this pilot study. The patient was right-handed and showed WC-specific symptoms, such as feeling difficulty in writing but not in other hand motor functions. The diagnosis was provided by a neurologist and physiatrist. Since the WC was not caused by secondary causes, such as drug use or head injury, the patient classified as primary dystonia patient. Moreover the WC in this study was categorized sporadic dystonia same as to general type of WC patients. The patient had been on medication for one year, but treatment was halted since the improvement of symptoms was not confirmed before this study started. Incidentally, the botulinum toxin injection for WC or focal hand dystonia is not covered by health care insurance and not practiced in Japan, so the patient had never received it.

The patient gave written informed consent for this study and the publication of individual data, which was approved by the local ethical committee of Asahikawa Medical University Hospital, and the study was conducted in accordance with the Declaration of Helsinki. The patient participated in the experiment twice a month over a 5 -month period, a total of 10 days. Every experiment was finished within 2 hours to avoid fatigue.

\section{Biosignal measurement}

We recorded 10-channel monopolar EEG and 3-channel surface EMG from the forearm during the experiment with $\mathrm{Ag} / \mathrm{AgCl}$ electrodes (diameter $9 \mathrm{~mm}$ ). To record scalp EEG, the electrodes were placed at C3 and C4, as designated according to the International 10/20 system, and $2.5 \mathrm{~cm}$ anterior, posterior, left, and right to $\mathrm{C} 3$ and $\mathrm{C} 4$, close to the hand representation area. All EEG channels were referenced to the right ear lobe. The ground electrode was positioned on the forehead. The EEGs were then converted to a reference-free form by a Laplacian algorithm [20] that used the set of the four neighbor electrodes. For electrode C3 (C4), these were anterior, posterior, left, and right to C3 (C4). The two Laplacian EEGs are called the left EEG (C3) and the right EEG (C4) in this study.

The surface EMG of the right hand (dominant hand) was recorded from the ECR muscle.

All biosignals were band-pass filtered between 2 and $1000 \mathrm{~Hz}$, and simultaneously digitized at $2400 \mathrm{~Hz}$ using a biosignal amplifier (g.USBamp, gtec, Austria). The EEG data were used online and were also stored on a personal computer for offline analysis.

\section{Assessment set}

In each daily assessment, we conducted EEG and EMG measurement during simple motor tasks both before and after 1-hour BCI training to assess the electrophysiological signal changes.

For each assessment, the patient was requested to perform the following: (1) tonic contraction of the right ECR muscle (tonic motor task) for $70 \mathrm{~s}$; (2) right hand extension with a rhythmic auditory cue 40 times, one every $2.5 \mathrm{~s}$ (repetitive motor task); and (3) write Japanese and Chinese characters with a ballpoint pen (writing task). 
During the tonic motor task, the participant maintained a constant and weak extension force with integrated EMG recorded from the right ECR muscle. Integrated EMG displayed for the patient was computed, rectifying and smoothing with a moving average with a window of $1 \mathrm{~s}$.

\section{Time-frequency representation}

In the EEG analysis, we drew ERD/ERS maps using the intertrial variance method [21-23]. The two Laplacian EEG data sets from the repetitive motor task were analyzed between 5 and $50 \mathrm{~Hz}$ in intervals of $2 \mathrm{~Hz}$. Trials were filtered with a 4th order Butterworth filter, and the ensemble average was subtracted from individual trials (total 40 trials). This operation reduces the contribution of phase-locked responses to the ERD/ERS quantification. The trials were squared and then averaged using a moving average window of $250 \mathrm{~ms}$ smoothed to estimate the power change in each frequency band. The reference interval for the relative power changes was between -1.5 and $-0.5 \mathrm{~s}$ from the onset of the EMG from the right ECR.

The statistical significance of the ERD/ERS values was determined by applying a t-percentile bootstrap algorithm [24] with a significance level of $1 \%$. Significant ERD/ERS data were shown as time-frequency maps.

\section{Cortico-muscular coherence analysis}

To access the linear involvement of cortical oscillations in motor unit synchronization, cortico-muscular coherence analysis was conducted on bilateral EEG and dystonic right EMG during the tonic motor task. The computation process was the same as in previous cortico-muscular coherence studies [25-27]. Following segmentation of the data stream into 70 segments of 1-s duration, the segment was windowed with a 1-s Hamming window (90\% overlap), and a Fourier transform was used. The segment length of 1 second leads to a $1-\mathrm{Hz}$ frequency resolution. The $99 \%$ confidence limit determined by Monte Carlo simulation [28] was approximately 0.017 .

\section{$\mathrm{BCl}$ paradigm}

During BCI training, a visual feedback monitor displayed a star as moving upward or downward based on the EEG features-related dystonic muscular contraction with an update rate of $16 \mathrm{~Hz}$. The same visual feedback method was employed in a previous study [8].

Using EEG time-frequency maps, we identified EEG frequency bands that reflected the cortical excitability of the sensorimotor cortex. We also confirmed the influence of the EEG oscillation in these frequency bands on the EMG of the forearm muscles using cortico-muscular coherence analysis. As visual feedback in the BCI intervention, the logarithms of the band power of EEG recorded from the bilateral hemispheres $\left(x_{1}\right.$ and $\left.x_{2}\right)$ were used for linear discriminant

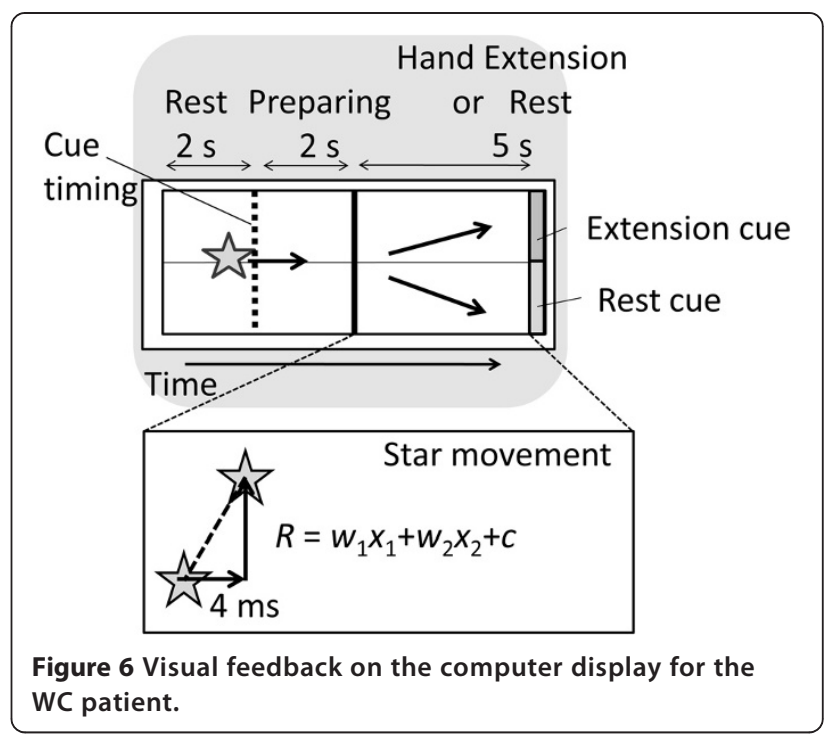

analysis (LDA) to detect the dystonia-specific power changes as feature values.

The LDA classifier parameters (weights $w_{1}$ and $w_{2}$ and a constant $c$ ) were calculated using the data of repetitive motor tasks in pre-BCI neurorehabilitation. The calculation was done based on cue-based 2 class wise classification, the same as a previous study [29], and we picked the LDA parameters that showed the best classification accuracy in 2 cross-fold validation.

The LDA classification result $(R)$ was converted to the online movement of a star in the Y-axis as visual feedback for the patient (Figure 6). The star's movement was calculated based on the following equation:

$$
R=w_{1} x_{1}+w_{2} x_{2}+C
$$

The displacement of the star, $R, x_{1}$, and $x_{2}$ calculated from the last 1-s data were updated every $4 \mathrm{~ms}(250 \mathrm{~Hz})$ in the PC processing. Due to the refresh rate of the PC screen, the actual refresh rate was approximately $60 \mathrm{~Hz}$. A positive/negative value of $R$ was translated into the representation of a right hand extension/resting, and into an upward/downward displacement of the star.

All analyses described above were carried out using MATLAB software (The MathWorks, US) with customdeveloped programs.

\section{Endnote}

${ }^{a}$ Short report including preliminary data, which was partly used in this study, nominated as one of top 10 reports for "The International Annual BCI Award," supported by g.tec, 2013.

\section{Competing interests}

The authors declare that they have no competing interests. 


\section{Authors' contributions}

$\mathrm{YH}$ made substantial contributions to the experimental conception and design, data acquisition, and data analysis and interpretation; JU was involved in drafting and revising the manuscript; and $M L, T O$, and $M M$ gave final approval of the revised manuscript for publication. All authors read and approved the final manuscript.

\section{Acknowledgements}

This work was supported by JSPS KAKENHI Grant Numbers, 24500568 and 25750197 and also resulted from "Brain-machine interface development" under the Strategic Research Program for Brain Sciences (SRPBS) by the Ministry of Education, Culture, Sports, Science and Technology of Japan (MEXT).

\section{Author details}

${ }^{1}$ Department of Electrical and Electronic Engineering, Kitami Institute of Technology, Hokkaido, Japan. ${ }^{2}$ Asahikawa Medical University Hospital, Hokkaido, Japan. ${ }^{3}$ Department of Rehabilitation Medicine I, School of Medicine, Fujita Health University, Aichi, Japan. ${ }^{4}$ Department of Rehabilitation Medicine, Keio University School of Medicine, Tokyo, Japan. ${ }^{5}$ Department of Biosciences and Informatics, Faculty of Science and Technology, Keio University, Kanagawa, Japan.

Received: 6 June 2014 Accepted: 28 August 2014

Published: 1 September 2014

\section{References}

1. Birch GE, Bozorgzadeh Z, Mason SG: Initial on-line evaluations of the LF-ASD brain-computer interface with able-bodied and spinal-cord subjects using imagined voluntary motor potentials. IEEE Trans Neural Syst Rehabil Eng 2002, 10:219-224

2. Kübler A, Nijboer F, Mellinger J, Vaughan TM, Pawelzik H, Schalk G, McFarland DJ, Birbaumer N, Wolpaw JR: Patients with ALS can use sensorimotor rhythms to operate a brain-computer interface. Neurology 2005, 64:1775-1777.

3. Ramos-Murguialday A, Broetz D, Rea M, Läer L, Yilmaz O, Brasil FL, Liberati G, Curado MR, Garcia-Cossio E, Vyziotis A, Cho W, Agostini M, Soares E, Soekadar S, Caria A, Cohen LG, Birbaumer N: Brain-machine interface in chronic stroke rehabilitation: a controlled study. Ann Neurol 2013, 74:100-108.

4. Daly JJ, Wolpaw JR: Brain-computer interfaces in neurological rehabilitation. Lancet Neurol 2008, 7:1032-1043.

5. Wang W, Collinger JL, Perez MA, Tyler-Kabara EC, Cohen LG, Birbaumer N, Brose SW, Schwartz AB, Boninger ML, Weber DJ: Neural interface technology for rehabilitation: exploiting and promoting neuroplasticity. Phys Med Rehabil Clin N Am 2010, 21:157-178.

6. Broetz D, Braun C, Weber C, Soekadar SR, Caria A, Birbaumer N: Combination of brain-computer interface training and goal-directed physical therapy in chronic stroke: a case report. Neurorehabil Neural Repair 2010, 24:674-679.

7. Caria A, Weber C, Brötz D, Ramos A, Ticini LF, Gharabaghi A, Braun C, Birbaumer N: Chronic stroke recovery after combined $\mathrm{BCl}$ training and physiotherapy: a case report. Psychophysiology 2011, 48:578-582.

8. Shindo K, Kawashima K, Ushiba J, Ota N, Ito M, Ota T, Kimura A, Liu M: Effects of neurofeedback training with an electroencephalogram-based brain-computer interface for hand paralysis in patients with chronic stroke: a preliminary case series study. J Rehabil Med 2011, 43:951-957.

9. Mukaino M, Ono T, Shindo K, Fujiwara T, Ota T, Liu M, Ushiba J: The efficacy of brain-computer interface-driven neuromuscular electrical stimulation for chronic paresis after stroke. J Rehabil Med. in press.

10. Fahn S, Bressman SB, Marsden CD: Classification of dystonia. Adv Neurol 1998, 78:1-10

11. Sheehy MP, Marsden CD: Writers' cramp-a focal dystonia. Brain 1982 105(Pt 3):461-480

12. Ridding MC, Sheean G, Rothwell JC, Inzelberg R, Kujirai T: Changes in the balance between motor cortical excitation and inhibition in focal, task specific dystonia. J Neurol Neurosurg Psychiatr 1995, 59:493-498.

13. Preibisch C, Berg D, Hofmann E, Solymosi L, Naumann M: Cerebral activation patterns in patients with writer's cramp: a functional magnetic resonance imaging study. J Neurol 2001, 248:10-17.

14. Kanovský $P$, Bares $M$, Streitová $H$, Klajblová $H$, Daniel $P$, Rektor I: Abnormalities of cortical excitability and cortical inhibition in cervical dystonia evidence from somatosensory evoked potentials and paired transcranial magnetic stimulation recordings. J Neurol 2003, 250:42-50

15. Toro C, Deuschl G, Hallett M: Movement-related electroencephalographic desynchronization in patients with hand cramps: evidence for motor cortical involvement in focal dystonia. Ann Neurol 2000, 47:456-461.

16. Tecchio F, Melgari JM, Zappasodi F, Porcaro C, Milazzo D, Cassetta E, Rossini PM: Sensorimotor integration in focal task-specific hand dystonia: a magnetoencephalographic assessment. Neuroscience 2008, 154:563-571.

17. Ushiyama J, Suzuki T, Masakado Y, Hase K, Kimura A, Liu M, Ushiba J: Between-subject variance in the magnitude of corticomuscular coherence during tonic isometric contraction of the tibialis anterior muscle in healthy young adults. J Neurophysiol 2011, 106:1379-1388.

18. Hashimoto Y, Ushiba J, Kimura A, Liu M, Tomita Y: Correlation between EEG-EMG coherence during isometric contraction and its imaginary execution. Acta Neurobiol Exp (Wars) 2010, 70:76-85.

19. Wissel J, Kabus C, Wenzel R, Klepsch S, Schwarz U, Nebe A, Schelosky L, Scholz U, Poewe W: Botulinum toxin in writer's cramp: objective response evaluation in 31 patients. J Neurol Neurosurg Psychiatr 1996, 61:172-175.

20. Hjorth B: An on-line transformation of EEG scalp potentials into orthogonal source derivations. Electroencephalogr Clin Neurophysiol 1975, 39:526-530.

21. Kalcher J, Pfurtscheller G: Discrimination between phase-locked and non-phase-locked event-related EEG activity. Electroencephalogr Clin Neurophysiol 1995, 94:381-384.

22. Solis-Escalante T, Müller-Putz GR, Pfurtscheller G, Neuper C: Cue-induced beta rebound during withholding of overt and covert foot movement. Clin Neurophysiol 2012, 123:1182-1190.

23. Hashimoto $Y$, Ushiba J: EEG-based classification of imaginary left and right foot movements using beta rebound. Clin Neurophysiol 2013, 124:2153-2160

24. Davison AC, Hinkley DV: Bootstrap Methods and Their Application Cambridge: University Press; 1997

25. Mima T, Hallett M: Electroencephalographic analysis of cortico-muscular coherence: reference effect, volume conduction and generator mechanism. Clin Neurophysiol 1999, 110:1892-1899.

26. Perez MA, Lundbye-Jensen J, Nielsen JB: Changes in corticospinal drive to spinal motoneurones following visuo-motor skill learning in humans. J Physiol 2006, 573:843-855.

27. Raethjen J, Govindan RB, Muthuraman M, Kopper F, Volkmann J, Deuschl G: Cortical correlates of the basic and first harmonic frequency of Parkinsonian tremor. Clin Neurophysiol 2009, 120:1866-1872.

28. McKeown MJ, Palmer SJ, Au WL, McCaig RG, Saab R, Abu-Gharbieh R: Cortical muscle coupling in Parkinson's disease (PD) bradykinesia. J Neural Transm Supp/ 2006, 70:31-40

29. Ron-Angevin R, Díaz-Estrella A: Brain-computer interface: changes in performance using virtual reality techniques. Neurosci Lett 2009, 449:123-127.

doi:10.1186/1471-2202-15-103

Cite this article as: Hashimoto et al: Functional recovery from chronic writer's cramp by brain-computer interface rehabilitation: a case report. BMC Neuroscience 2014 15:103.

\section{Submit your next manuscript to BioMed Central and take full advantage of:}

- Convenient online submission

- Thorough peer review

- No space constraints or color figure charges

- Immediate publication on acceptance

- Inclusion in PubMed, CAS, Scopus and Google Scholar

- Research which is freely available for redistribution 